\title{
The Relationship Between Repeated-Sprint Ability, Aerobic Capacity, and Oxygen Uptake Recovery Kinetics in Female Soccer Athletes
}

\author{
by \\ Bruno Archiza', Daniela K. Andaku1, Thomas Beltrame ${ }^{1}$, Cleiton A. Libardi², \\ Audrey Borghi-Silva ${ }^{1}$
}

\begin{abstract}
This study investigated the relationship between repeated-sprint ability, aerobic capacity, and oxygen uptake kinetics during the transition between exercise and recovery (off-transient) in female athletes of an intermittent sport modality. Eighteen professional soccer players completed three tests: 1) a maximal incremental exercise test; 2) a constant speed time-to-exhaustion test; and 3) a repeated-sprint ability test consisting of six 40-m sprints with $20 \mathrm{~s}$ of passive recovery in-between. Correlations between time-to-exhaustion, repeated-sprint ability, and oxygen uptake kinetics were calculated afterwards. The level of significance was set at $p<0.05$. A performance decrement during repeated-sprint ability was found to be related to: 1) time-to-exhaustion (e.g., exercise tolerance; $r=-0.773, p<0.001$ ); 2) oxygen uptake recovery time ( $r=0.601, p=0.008)$; and 3) oxygen uptake mean response time of recovery $(r=0.722$, $p<0.001)$. Moreover, the best sprint time $(r=-0.601, p=0.008)$ and the mean sprint time $(r=-0.608, p=0.007)$ were found to be related to maximal oxygen uptake. Collectively, these results reinforce the relation between oxygen uptake kinetics and the ability to maintain sprint performance in female athletes. These results may contribute to coaches and training staff of female soccer teams to focus on training and improve their athletes' aerobic capacity and recovery capacity to improve intermittent exercise performance.
\end{abstract}

Key words: oxygen uptake kinetics, sprinting ability, intermittent modality, women soccer, women athletes.

\section{Introduction}

Most intermittent-like sport modalities (e.g., team and racket sports) are characterized by short periods of high-intensity sprints, followed by periods of active and passive recovery. For example, the total sprint distance covered by players in a soccer match is an important factor related to overall performance (Di Salvo et al., 2009; Datson et al., 2019). Therefore, repeatedsprint ability (RSA) tests have been largely used as an important index of intermittent sport performance (Wragg et al., 2000), and to track the effectiveness of a training programme (Impellizzeri et al., 2008). On the other hand, Taylor et al. (2016) proposed that RSA should be viewed as a means of fitness training as opposed to a means of fitness assessment. In fact, a recent review from the same group has shown that repeated-sprints training is beneficial for speed, leg-power and strength, high-intensity running performance, and aerobic capacity of team sport athletes (Taylor et al., 2015). Similarly, other studies have shown a positive correlation between RSA and aerobic capacity (e.g., maximal oxygen uptake, $\mathrm{VO}_{2 m a x}$ ) (Aziz et al., 2000; Bishop and Spencer, 2004). Thus, those athletes with higher $\mathrm{VO}_{2 \max }$ values demonstrated better repeated sprint performance.

Indeed, this relationship may impact soccer performance directly, for example distance covered during a match, the number of involvements with the ball, and the possibility to

1 - Department of Physical Therapy, Federal University of São Carlos, São Carlos, São Paulo, Brazil.

2 - Department of Physical Education, Federal University of São Carlos, São Carlos, São Paulo, Brazil. 
influence a match outcome (Helgerud et al., 2001). Note that this relationship is strictly related to the RSA protocol type, which may vary in the number of sprints, movement direction change, passive or active recovery, and test duration (Dupont et al., 2010). Another important factor is the recovery time between sprints. In other words, how quickly an athlete is physiologically ready to perform a successive sprint after maximal effort, which is measured using a performance decrement or a fatigability index during the RSA test (Bishop et al., 2011). As previously seen, $\mathrm{VO}_{2}$ kinetics may also be used as a determinant of fatigability and exercise performance (Jones and Burnley, 2009; Dupont et al., 2010). Significantly faster $\mathrm{VO}_{2}$ kinetics during both onset of exercise and recovery was attributed to positive adaptations to endurance exercise training (Hagberg et al., 1980), due to optimized convective oxygen transport, tissue oxygen extraction, blood lactate removal, and a more significant phosphocreatine $(\mathrm{PCr})$ resynthesis rate in the RSA context (Børsheim and Bahr, 2003). Dupont et al. (2005) showed that the ability of an athlete to maintain sprint performance depended on recovery mechanisms, thus any relationship found between $\mathrm{VO}_{2}$ kinetics at the onset of exercise (on-transient) and the RSA performance decrement (RSADEC) would be a consequence rather than a cause. Hence, the analysis of $\mathrm{VO}_{2}$ kinetics from exercise to recovery (off-transient) would better reflect recovery factors associated with RSADEc. However, these premises were tested in male-only or mixed-sex samples.

It is broadly known that the selection and utilization of metabolic substrates during exercise are different between males and females. Indeed, muscle morphology and histology, substrate utilization patterns and strategies (e.g., cellular mechanisms), as well as hormonal regulation are some of the influential factors that differ between sexes during high-intensity exercise (Ruby and Robergs, 1994). However, the discrepancies between sexes regarding the mentioned factors tend to narrow as the level of cardiorespiratory fitness and aerobic capacity increases to the level of highly trained individuals and/or professional athletes (Ruby and Robergs, 1994). However, at present and to the best of our knowledge, the effects of oxygen uptake recovery kinetics on sprinting performance of female soccer athletes are still unclear. Thus, the purpose of this study was to verify the relationship between RSA variables (especially those related to recovery characteristics) vs. aerobic capacity and $\mathrm{VO}_{2}$ recovery kinetics of female soccer athletes. We hypothesized that participants with better RSA performance would also have a better aerobic capacity and faster $\mathrm{VO}_{2}$ recovery kinetics. If our hypothesis is true, future female soccer training programs should focus on aerobic capacity and $\mathrm{VO}_{2}$ recovery variables to obtain better sprinting performance of athletes.

\section{Methods}

\section{Participants}

Twenty-five professional female players of a Brazilian premier soccer league team were initially recruited; of those, 7 were withdrawn from the study for the following reasons: team change $(n=3)$, orthopaedic injury $(n=2)$, and declined to participate $(n=2)$. In total, 18 athletes completed all procedures; however, the goalkeepers $(n=2)$ were removed from the final analysis given their differences in physiological/performance requirements and training. Thus, 16 athletes $(22 \pm 4$ years, $163 \pm 6$ $\mathrm{cm}, 56.6 \pm 7.1 \mathrm{~kg}$ ) were included in the final analysis. Our participants included 3 defenders, 4 fullbacks, 4 midfielders (back, central, or wide midfield), and 5 centre forwards. As inclusion criteria, all athletes should have been training in a professional level team for at least 12 months and have no injuries during the last 6 months of the study. Written informed consent was obtained from all participants prior to testing. The experimental design was approved by the Research Ethics Committee at the local institution (approval number 735.586/2014) and conformed to the Declaration of Helsinki for human experimentation. This study was registered in a national public repository (code RBR-7K6PFB).

\section{Study Design}

To address the main question of the study and test our hypothesis, the study design was composed of three testing days separated by at least 48 hours. Day 1 consisted of anthropometric measurements, familiarization with the procedures, a maximal incremental exercise (CPX) test to determinate aerobic capacity (maximal or peak $\mathrm{VO}_{2}$ ) and maximal aerobic speed on a treadmill (details below). On day 2, participants 
performed a constant speed time-to-exhaustion (TTE) test using the maximal aerobic speed assessed on day 1 . Subsequently, on day 3 , the RSA test was performed on a soccer grass field. All tests were performed at the same time of day and athletes were instructed to have a light meal 2 hours prior to each test and to avoid any stimulant beverages. Off-transient $\mathrm{VO}_{2}$ kinetic values were calculated using recovery data from the TTE test, which reflects a high-intensity steady-state exercise and the overall aerobic condition of each athlete. Lastly, correlations between $\mathrm{VO}_{2}$ off-transient kinetics and RSA test variables were performed to test our hypothesis.

\section{Measures and Procedures}

On day 1, participants performed the CPX test on a treadmill (Super ATL, Inbramed, Brazil) programmed to simulate outdoor running, as the inclination was fixed at $1 \%$ during the entire test (Jones and Doust, 1996). Initially, the participant remained standing for 5-min to collect resting data followed by a 3-min warm-up at $4 \mathrm{~km} \cdot \mathrm{h}^{-1}$. After warming-up, speed increased to $8 \mathrm{~km} \cdot \mathrm{h}^{-1}$ for one minute with subsequent stage increments of 1 $\mathrm{km} \cdot \mathrm{h}^{-1}$ per minute until exhaustion. Immediately after test cessation, $1 \mathrm{~min}$ of active cool-down ( $0 \%$ inclination at $4 \mathrm{~km} \cdot \mathrm{h}^{-1}$ ) was followed by a 5 -min standing passive rest on the treadmill. A heart rate (HR) monitor (Polar S810i, Polar Electro, Finland) was used to obtain the beat-by-beat HR during the entire test. Respiratory gas exchange was recorded breath-by-breath using a gas analyser (Oxycon Mobile, Jaeger, Germany). Maximal values were considered and obtained by averaging the last $30 \mathrm{~s}$ of exercise of each variable and prediction values were calculated accordingly (Esco et al., 2014; Myers et al., 2017).

High-intensity constant speed TTE tests are vastly used as a measurement of exercise tolerance (Welch et al., 2018). The TTE test consisted of a 5-min passive rest followed by a 3min warm-up at $4 \mathrm{~km} \cdot \mathrm{h}^{-1}$ with the treadmill fixed at $1 \%$ inclination. After the warm-up, the speed was quickly increased to the maximal aerobic speed assessed on day $1(100 \%$ speed of the last completed stage of the CPX protocol) and remained fixed until exhaustion, after which a 6min passive recovery was granted to collect offtransient kinetics data. During the entire test, participants were verbally encouraged, especially as the individual approached visible exhaustion.
Respiratory gas exchange and $\mathrm{HR}$ data were collected and monitored during the entire test. Additionally, arterialized blood samples from the earlobe puncture were collected at rest and at peak exercise intensity. Concentrations of blood lactate $\left(\left[\mathrm{Lac}^{-}\right] \mathrm{B}\right)$ were measured via an electroenzymatic method (YSI 2300 Stat Plus, YSI Inc., USA) following manufacturer's instructions. The TTE test was re-applied in 8 of the 18 athletes to generate test-retest reliability data (coefficient of variation $=2.9 \%$ for $\mathrm{VO}_{2}$ and $4.8 \%$ for TTE) .

Following this, breath-by-breath $\mathrm{VO}_{2}$ data related to the recovery period of the TTE test $\left(\mathrm{VO}_{2}\right.$ off-transient) were exported and fitted by a monoexponential function (equation 1) after being interpolated and filtered as described elsewhere (Beltrame and Hughson, 2017). The lowest sum of squared residuals was used to select the best equation variables that better represented the $\mathrm{VO}_{2}$ kinetics response during the resting transition (Beltrame and Hughson, 2017).

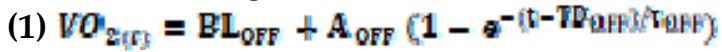

where, $\mathrm{BL}_{\alpha \mathrm{FF}}$ is the mean $\mathrm{VO}_{2}$ of the last $60 \mathrm{~s}$ before exercise cessation; $\mathrm{A}_{0 \mathrm{~m}}$ is the $\mathrm{VO}_{2}$ steadystate amplitude of the changes from the $\mathrm{BL}_{\propto \mathrm{FB}}$ during the recovery period; $\mathrm{TD}_{\mathrm{WF}}$ is the time delay of the exponential function after exercise cessation; and $\tau_{0 \mathrm{gF}}$ is the time constant of the exponential function during the recovery where lower values indicate faster $\mathrm{VO}_{2}$ off-transient kinetics. The quality of the data fitting was assessed by visual analysis of residuals and the degree of linearity between the fitted function and experimental data (Keir et al., 2016). In addition, mean response time (MRToff) was calculated as

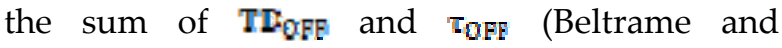
Hughson, 2017). A single-subject representative response of $\mathrm{VO}_{2}$ off kinetics is illustrated in Figure 1. Reliability of $\mathrm{VO}_{2}$ kinetics analysis was calculated by two blinded investigators (coefficient of variation $=5.6 \%$ ).

The RSA test used in this study was previously validated to assess male soccer players performance and it consisted of $6 \times 40 \mathrm{~m}(20 \mathrm{~m}+$ $180^{\circ}$ turn $+20 \mathrm{~m}$ ) shuttle sprints separated by $20 \mathrm{~s}$ of passive recovery (Impellizzeri et al., 2008; Rampinini et al., 2007). At least 48 hours later from day 2, athletes were instructed to come to the soccer field in their usual training clothes, shoes, and equipment. After a 10-min warm-up of mild running ( $\sim 65 \%$ maximal $\mathrm{HR})$, participants 
performed 3 submaximal sprints in order to become familiarized with the procedure. Afterwards, each athlete performed a single maximal sprint to provide a criterion time followed by a 5-min rest interval. Participants then performed 6 consecutive sprints, with a $20 \mathrm{~s}$ passive recovery in-between. With accuracy to $\mathrm{ms}$, the exact time of each sprint was recorded by a photocell system (Speed Test Fit, Cefise, Brazil). If the time of the first sprint was greater than $2.5 \%$ of the criterion time, the sprints sequence was interrupted and the participant rested for another 5 -min and then repeated the set of 6 sprints (Rampinini et al., 2007). The RSAdec was calculated with the following formula (equation 2):

\section{(2) $\left.\mathrm{BSA}_{\mathrm{DEC}}=\left[\mathrm{CBSA}_{\mathrm{MEAN}} / \mathrm{BSA}_{\mathrm{BEST}}\right) \times 100\right]-100$}

where RSAmEAN represents the mean RSA performance time for all sprints, and RSAвEST represents the best sprint time (fastest sprint). This method was previously reported as reliable and valid for quantifying the overall fatigue and recovery index (Glaister, 2008; Rampinini et al., 2007) during RSA tests. The total time of RSA (RSAтотAL) was also calculated by the sum of each sprint time. As the TTE test, RSA tests were reapplied in 10 of the 18 athletes to generate testretest reliability data (coefficient of variation $=$ $2.6 \%$ for RSAmEAn and $4.1 \%$ for RSAtotal).

\section{Statistical Analysis}

After verifying data distribution by the Shapiro-Wilk test, a t-test was applied to verify possible differences between CPX and TTE tests. Subsequently, the Pearson correlation matrix was created to verify the correlation coefficient $(r)$ between all TTE, RSA, and $\mathrm{VO}_{2}$ off-transient kinetic variables. The level of significance was set at $p<0.05$ and, when significant, $r$ was interpreted as: very small $(r=0-0.19)$, small $(r=0.20-0.49)$, moderate $(r=0.50-0.69)$, high $(r=0.70-0.89)$ or very high $(r=0.90-1.00)$ correlation (Evans, 1996). All analyses were performed using the GraphPad Prism version 6.01 (GraphPad Software Inc., La Jolla, CA, USA) and SigmaPlot version 12.0 (Systat Software Inc., San Jose, CA, USA) software packages. Results are expressed as means $\pm \mathrm{SD}$ unless otherwise noted.

\section{Results}

Maximal incremental and constant load exercise TTE tests results are shown in Table $\mathbf{1 .}$
There was a significative difference in $\mathrm{VO}_{2}$ values between the CPX and TTE tests. However, all participants presented $\mathrm{VO}_{2}$ values within prediction in both tests, moreover, they reached $>$ $90 \%$ of their peak $\mathrm{VO}_{2}$ during the TTE test, as well as maximal effort evidenced by the HR (even presenting statistical difference, both tests reached levels above $100 \%$ of the maximal predicted HR) and RER (Weisman et al., 2003). Expectedly, values of peak $\left[\mathrm{Lac}^{-}\right]_{\text {в }}$ were higher after the TTE compared to the CPX test, albeit not presenting statistical significance.

Regarding RSA test results, all sprint times, RSAbest, RSAmean, RSAtotal, and RSAdec data are presented in Table 2. Best performance times were achieved either in the first or the second sprint for all athletes. In addition, all athletes were able to match the criterion time in their first RSA test attempt.

With respect to the relationship between TTE and RSA testing, a high negative correlation between TTE and RSADEC $\left(r=-0.783, r^{2}=0.613, p<\right.$ $0.001)$, a very high positive correlation between the peak HR and RSAdec $\left(r=0.922, r^{2}=0.849, p<\right.$ $0.001)$, and a high positive correlation between peak [Lac ${ }^{-}$] and RSAdec $\left(r=0.710, r^{2}=0.503, p=\right.$ 0.002) were found (Figures 2A, 2B, and 2C, respectively). In addition, a moderate negative correlation between $\mathrm{VO}_{2}$ and RSAвEST $\left(r=-0.587, r^{2}\right.$ $=0.345, p=0.016)$, and between $\mathrm{VO}_{2}$ and RSAMEAN $\left(r=-0.574, r^{2}=0.329, p=0.020\right)$ were found (Figures 2D and 2E, respectively). All other variables from TTE $\left(\mathrm{VCO}_{2}, V_{\mathrm{E}}, \mathrm{RER}\right.$, and rest $\left[\mathrm{Lac}^{-}\right] \mathrm{B}$ ) and RSA testing (RSABest, RSAmean, RSAtotaL, and RSADEc) did not show significant correlations $(p>0.05)$ between each other.

With regard to the relationship between $\mathrm{VO}_{2}$ off-transient kinetics and the RSA performance decrement, a moderate positive correlation between $\tau_{\text {JHF }}(57.7 \pm 8.3 \mathrm{~s})$ and RSADEC $\left(r=0.578, r^{2}=0.334, p=0.019\right)$ was found, suggesting the longer the $\tau$ during recovery, the greater RSADEC; similarly, a moderate positive correlation between MRToff $(67.9 \pm 8.6 \mathrm{~s})$ and RSADEC $\left(r=0.693, r^{2}=0.480, p=0.002\right)$ was observed, indicating the longer the MRT during recovery, the greater the RSADEC (Figures $3 \mathbf{A}$ and 3B, respectively). $\mathrm{BL}_{\mathrm{OFF}}\left(1951.6 \pm 231.2 \mathrm{ml} \cdot \mathrm{min}^{-}\right.$ $\left.{ }^{1} \cdot \mathrm{kg}^{-1}\right)$ and $\mathbf{T D}_{\text {QFF }}(10.2 \pm 4.8 \mathrm{~s})$ did not present significant correlation $(p>0.05)$ vs. RSA variables (RSAbest, RSAmeAn, RSAtotal, and RSAdec). 
Table 1

Maximal incremental exercise (CPX) and constant load time-to-exhaustion (TTE) test data

\begin{tabular}{|c|c|c|c|}
\hline & CPX & TTE & $p$ \\
\hline TTE (s) & - & $259.6 \pm 97.9$ & - \\
\hline Peak HR (beats· min $^{-1}$ ) & $185.1 \pm 7.8$ & $179.5 \pm 7.3$ & .004 \\
\hline HR (\% predicted) & $104.6 \pm 5.1$ & $101.6 \pm 4.9$ & .151 \\
\hline Speed $\left(\mathrm{km} \cdot \mathrm{h}^{-1}\right)$ & - & $15.0 \pm 1.5$ & - \\
\hline $\mathrm{VO}_{2}\left(\mathrm{ml} \cdot \mathrm{min}^{-1} \cdot \mathrm{kg}^{-1}\right)$ & $42.9 \pm 6.8$ & $38.9 \pm 3.2$ & .014 \\
\hline $\mathrm{VO}_{2}\left(\mathrm{ml} \cdot \mathrm{min}^{-1}\right)$ & $2357.6 \pm 215.2$ & $2221.4 \pm 252.3$ & .014 \\
\hline$V \mathrm{O}_{2}(\%$ peak) & - & $94.2 \pm 11.4$ & - \\
\hline$V_{C O}\left(\mathrm{ml} \cdot \mathrm{min}^{-1}\right)$ & $2834.2 \pm 263.5$ & $2798.2 \pm 305.2$ & .502 \\
\hline$V_{\mathrm{E}}\left(1 \cdot \mathrm{min}^{-1}\right)$ & $94.6 \pm 17.1$ & $92.1 \pm 15.9$ & .083 \\
\hline RER & $1.2 \pm 0.1$ & $1.3 \pm 0.1$ & .162 \\
\hline $\operatorname{Rest}\left[\mathrm{Lac}^{-}\right]_{\mathrm{B}}\left(\mathrm{mmol} \cdot l^{-1}\right)$ & $1.3 \pm 0.4$ & $1.4 \pm 0.5$ & .839 \\
\hline Peak [ $\left.\mathrm{Lac}^{-}\right]_{\text {в }}\left(\mathrm{mmol} \cdot l^{-1}\right)$ & $6.4 \pm 2.3$ & $8.1 \pm 1.9$ & .346 \\
\hline
\end{tabular}

Data are presented as mean $\pm S D$. HR, heart rate; $\mathrm{VO}_{2}$, oxygen uptake; $\mathrm{VCO}_{2}$, carbon dioxide production; $V_{E}$, ventilation; $R E R$, respiratory exchange ratio; $\left[\mathrm{Lac}^{-}\right]_{B}$, blood lactate concentration.

Table 2

\begin{tabular}{lc}
\multicolumn{3}{c}{ Repeated-sprint ability (RSA) test data } \\
\hline Sprint number & $7.31 \pm 0.34$ \\
$1(\mathrm{~s})$ & $7.50 \pm 0.41$ \\
$2(\mathrm{~s})$ & $7.75 \pm 0.35$ \\
$3(\mathrm{~s})$ & $7.86 \pm 0.41$ \\
$4(\mathrm{~s})$ & $8.03 \pm 0.38$ \\
$5(\mathrm{~s})$ & $8.11 \pm 0.38$ \\
$6(\mathrm{~s})$ & $7.31 \pm 0.34$ \\
RSAвеSт (s) & $7.76 \pm 0.35$ \\
RSAмеAN (s) & $46.56 \pm 2.11$ \\
RSAтотаL (s) & $6.22 \pm 1.75$ \\
RSADEс (\%)
\end{tabular}

Data are presented as mean $\pm S D$. RSABEST, best time on a single sprint;

RSAMEAN, mean performance time; RSATOTAL, total sprints time;

$R S A D E C$, percentage of performance decrement throughout the test. 


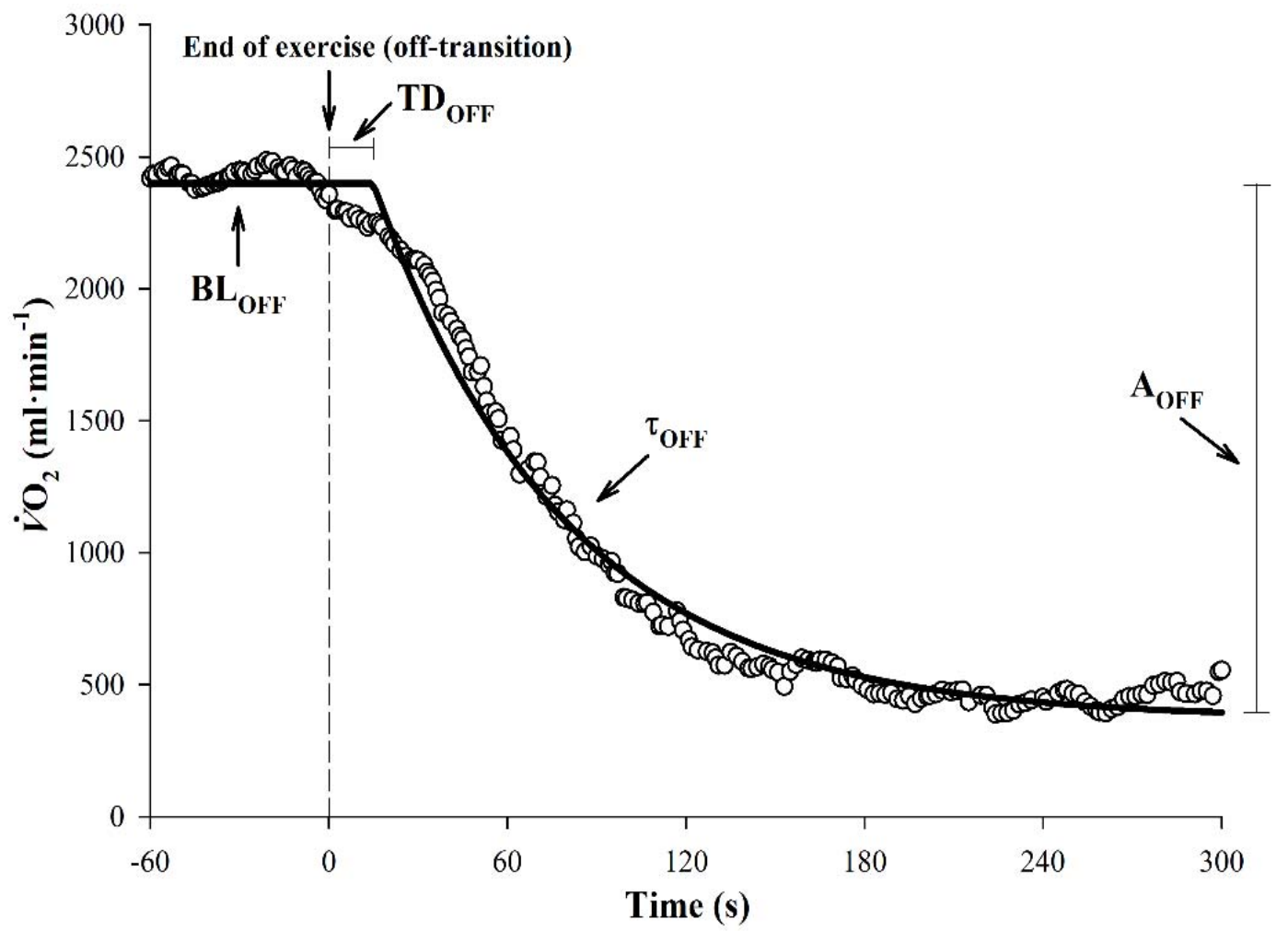

Figure 1

A representative figure from one participant depicting the oxygen uptake $\left(\mathrm{VO}_{2}\right)$ response during the recovery period (off-transient kinetics). Data were fitted by a mono-exponential function with variables also presented in the figure (see equation 2 in the text). The time zero represents exercise cessation. Mean baseline $\left(B E_{Q F F}\right)$, time delay $\left(T D_{W F}\right)$, time constant of the exponential function $\left(\tau_{O F F}\right)$, and the steady-state amplitude $\left(A_{O F F}\right)$ values of $\mathrm{VO}_{2}$ off-transient kinetics. 

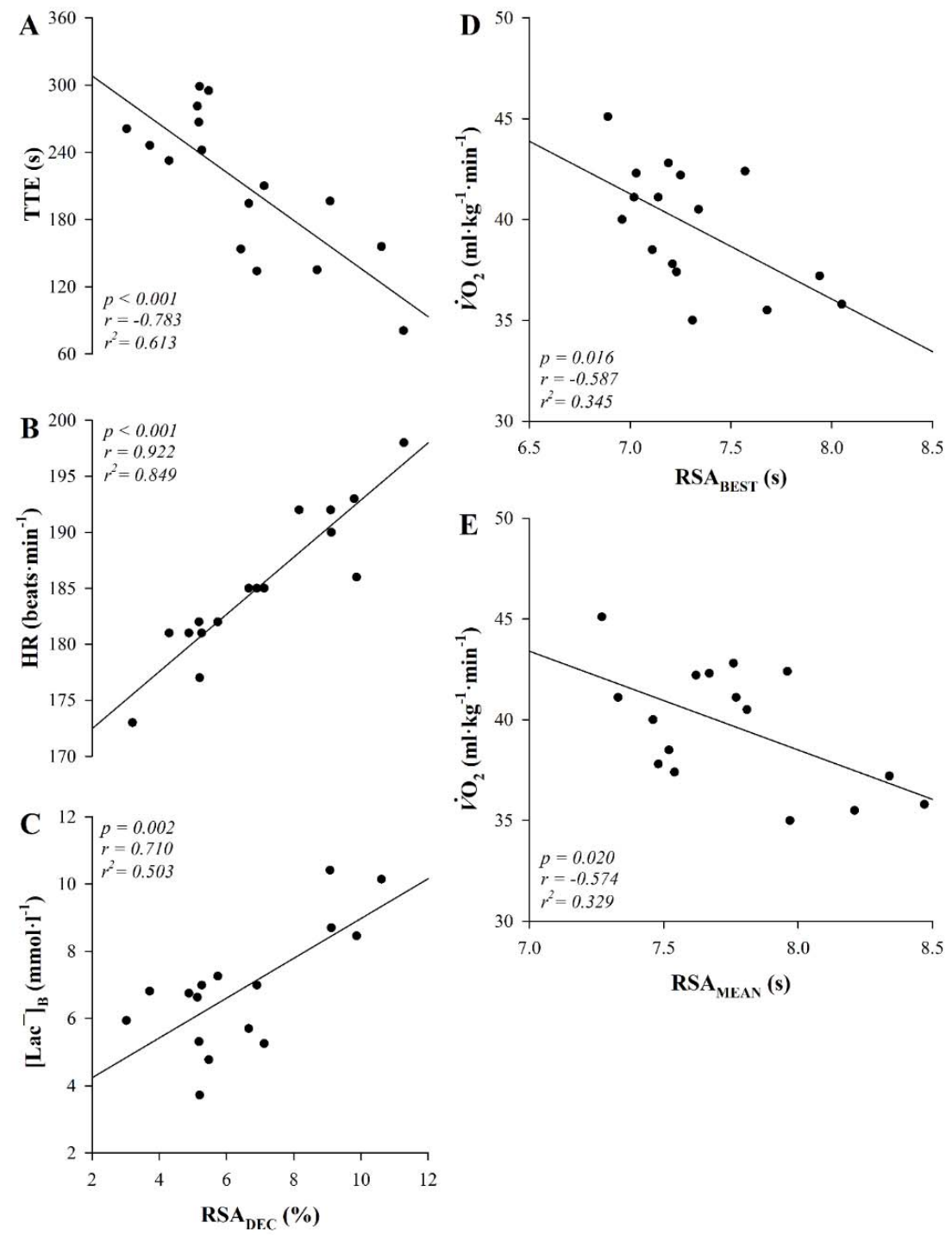

Figure 2

The relationship between constant load time-to-exhaustion (TTE) exercise and repeated-sprint ability (RSA) test data. Heart rate (HR), peak blood lactate concentration ([ $\left.\left.\mathrm{Lac}^{-}\right]_{B}\right)$, oxygen uptake $\left(\mathrm{VO}_{2}\right)$, percentage of performance decrement throughout RSA test (RSADEC), best sprint time (RSABEST), and mean sprint time (RSAMEAN). 

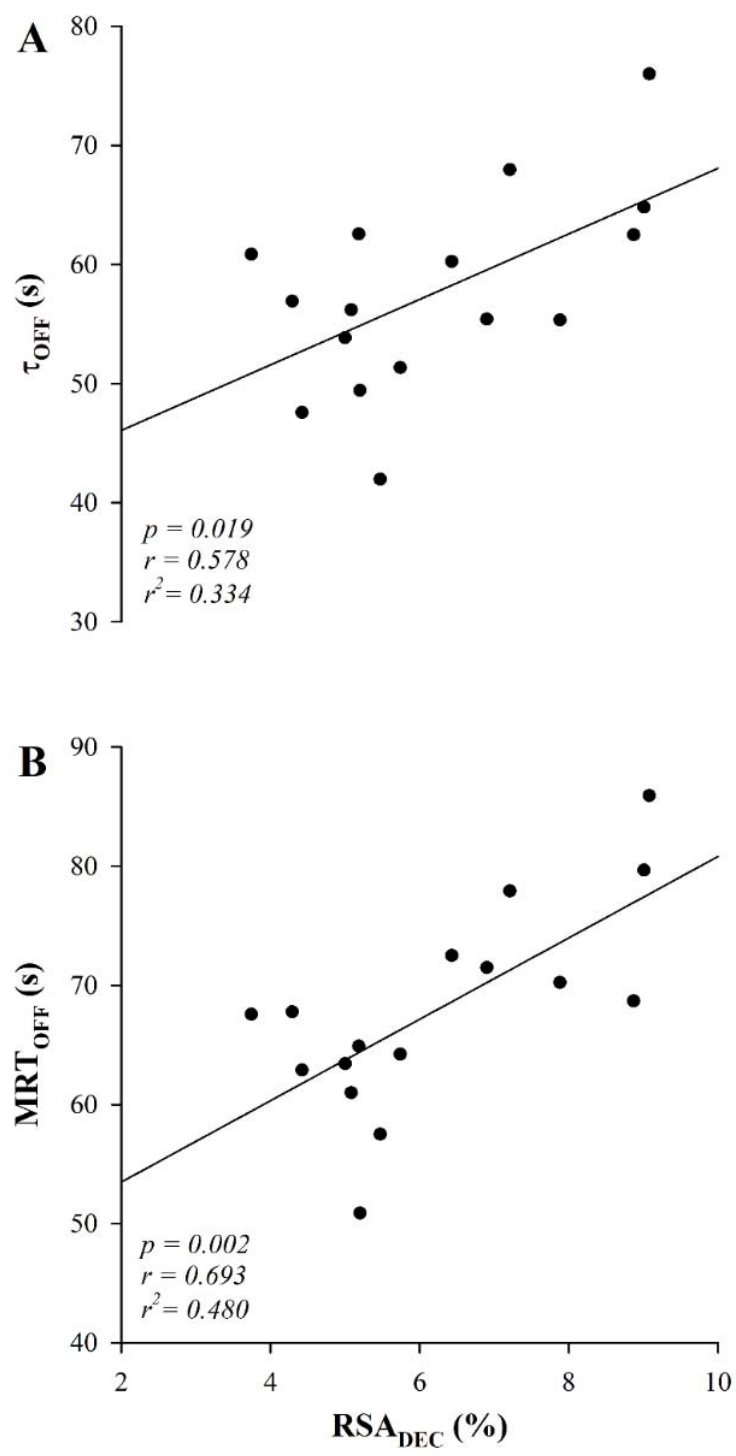

Figure 3

Relationship between oxygen uptake response during the recovery period (off-transient kinetics) and the repeated-sprint ability (RSA) test. Time constant of the exponential function ( $\tau_{O F F}$ ), mean response time (MRTofF), and percentage of performance decrement throughout RSA test (RSADEC). 


\section{Discussion}

The main results of this study are twofold: 1) a negative correlation between highintensity exercise tolerance (e.g., TTE) and the RSA performance decrement, and a positive correlation between the peak $\mathrm{HR}$ and blood lactate concentration with the RSA performance decrement; also, a negative correlation between $\mathrm{VO}_{2}$ and overall RSA performance (e.g., RSAвEST and RSAmEAN); and 2) a positive correlation between the time-based recovery of $\mathrm{VO}_{2}$ and $\mathrm{VO}_{2}$ mean response time of recovery with the RSA test performance decrement in female soccer players. That is to say, those players who presented higher aerobic capacity and faster oxygen kinetics recovery after high-intensity exercise, also presented a better overall RSA performance and greater recovery performance during the RSA test, respectively. We thus suggest that the RSA test could be used as an effective indicator of exercise performance in female soccer players.

The RSA is a specific test which mimics a situation of intermittent exercise, and has been used as an important tool to measure sprint ability and the recovery index in athletes of this specific exercise modality (Datson et al., 2019; Rampinini et al., 2007). In the present study, evaluation of the recovery index during RSA was reported as RSADEC, which is an indicator of athletes' ability to maintain sprint performance and has been associated with oxygen and $\mathrm{PCr}$ replenishment in short periods of rest between sprint efforts (Welch et al., 2018). Moreover, the accumulation of [ $\left.\mathrm{Lac}^{-}\right]_{\mathrm{B}}$ also has a relation with RSADEC and RSA overall performance. It has been shown that poorer sprint ability is related to low muscle PCr (Bogdanis et al., 1995), while potassium within muscle tissue (Nordsborg et al., 2015) and inorganic phosphate accumulate (Allen et al., 2008) during high-intensity sprint activity. Likewise, the ability and velocity of the system to recover these metabolic issues is related to better sprint performance (Dupont et al., 2005). It has been shown that the analysis of $\mathrm{VO}_{2}$ off-transient kinetics is related to recovery time during intermittent tests (Dupont et al., 2005). Nonetheless, the results of this study suggest that faster $\mathrm{VO}_{2}$ off-transient kinetics are related to a faster recovery of variables contributing to overall fatigue.

Previous research has shown that low $\mathrm{PCr}$ concentration within the muscles may impair sprint ability (Bogdanis et al., 1995). Likewise, high concentrations of inorganic phosphate and potassium also play a major role in sprinting ability and recovery (Allen et al., 2008). Unsurprisingly, along with high blood lactate concentration, these are the metabolites responsible for activation of central and peripheral neural ways responsible for effort and fatigue perception (Romer and Polkey, 2008). Albeit, this is only one factor contributing to peripheral and central fatigue; $\mathrm{PCr}$ resynthesis plays a major role in the maintenance of performance (Glaister, 2008). Indeed, some studies have demonstrated that oxygen availability is linked to $\mathrm{PCr}$ replenishment in the muscle, especially during the recovery period, when there are no muscle active contractions and blood flow and oxygenation are maximized (Harris et al., 1976; Haseler et al., 1999). Thus, it is plausible to assume that faster oxygen kinetics would ameliorate these symptoms and improve exercise performance. To this end, we found a significant positive correlation between $\left[\mathrm{Lac}^{-}\right]_{\mathrm{B}}$ and RSADEC in this study, representing that those who presented lower lactate accumulation during a severe-intensity exercise were those with a lower RSA performance decrement, faster offtransient $\mathrm{VO}_{2}$ kinetics, and higher TTE.

Interestingly, we found a significant correlation between the time-based recovery of $\mathrm{VO}_{2}$ and $\mathrm{VO}_{2}$ mean response time of recovery with the RSA decrement in female soccer players. Dupont et al. (2010) also found a strong correlation between $\mathrm{VO}_{2}$ off-transient kinetics and a lower RSADEC in male soccer players; those authors suggested that a faster $\mathrm{VO}_{2}$ recovery time in severe-intensity exercise was linked to a better ability to maintain sprint performance. Other studies confirmed these results, linking aerobic capacity to RSAdec (Aziz et al., 2000). Moreover, as presented previously, better performance related to faster oxygen uptake kinetics was previously found in middle- and long-distance runners (Kilding et al., 2006), but not in intermittent-like modalities of an only-women sample. Indeed, this is the first study to show a significant positive correlation between $\mathrm{VO}_{2}$ offtransient kinetics and RSADEC in a cohort of female soccer players.

It has been suggested that muscle fibres 
with higher mitochondrial volume density have an important influence on $\mathrm{VO}_{2}$ off-transient kinetics (Barstow et al., 1996); thus muscle fibre distribution would influence oxygen uptake kinetics. Muscle fibres type II are recognized to have higher mitochondrial volume density when compared to type I fibres (Meyer et al., 1985), and to present a different metabolism of cellular respiration, quantitatively and qualitatively, which is linked to the $\mathrm{PCr}$ usage and replenishment mechanism (Kushmerick et al., 1992). In this study, we did not measure muscle fibre type volume, thus future investigations that include this methodology would clarify the relationship between the muscle fibre type volume and oxygen kinetics metabolism.

In contrast, one study did not find a significant influence of training volume and $\mathrm{VO}_{2}$ on-transient kinetics while reporting a strong relation between training volume and $\mathrm{VO}_{2}$ offtransient kinetics (Kilding et al., 2006). In this instance, the authors evaluated middle- and longdistance runners who had different weekly training volumes. In this context, it would be important to identify the best training strategies in order to reach gains in $\mathrm{VO}_{2}$ off-transient kinetics. This has been questioned before, though remains unclear; authors have found conflicting evidence as to the ideal training strategy to influence $\mathrm{VO}_{2}$ on-transient kinetics (Bailey et al., 2009; Murias et al., 2010). Future studies are necessary to reveal what training strategies are most efficient in influencing $\mathrm{VO}_{2}$ off-transient kinetics in intermittent modalities.

There are some limitations in the present study that we must address. Firstly, we did not measure metabolic and respiratory variables during the RSA test; these data would elucidate the mechanisms present during this type of exercise and would help understand the link between aerobic capacity and $\mathrm{VO}_{2}$ kinetics in intermittent activities. Secondly, we did not assess muscle fibre distribution via biopsy; this technique would give us a glimpse into muscle physiology related to fibre distribution and mitochondrial density, and their relation with $\mathrm{VO}_{2}$ kinetics in intermittent modalities in this population. Thirdly, $\mathrm{VO}_{2}$ off-kinetics method has an important limitation which refers to high variability in the analysis of $\mathrm{VO}_{2}$ once it is based on a breath-by-breath system and may lead to a low signal-to-noise ratio. On the other hand, we used specific filters (moving average, exclusion of values greater than $4 \mathrm{SD}$ ) in this study to maintain variables within the $95 \%$ confidence interval (Rossiter et al., 2002). Fourthly, no measures were taken during a soccer match play (e.g., distance covered, number of sprints); these measurements would help to better characterise our participants' training and athletic level. Lastly, despite being validated and well described (Rampinini et al., 2007), we have used only one specific test to assess intermittent exercise performance; we consider that other tests would help us understand more about the relationship between $\mathrm{VO}_{2}$ kinetics and intermittent modalities.

In summary, the results of the present study showed a significant relationship between $\mathrm{VO}_{2}$ off-transient kinetics (Toff and MRTоғF) and a high-intensity constant speed TTE test as well as the decrement of performance during the RSA test in female soccer players. These results suggest that those athletes presenting higher oxygen uptake recovery time (e.g., faster $\mathrm{VO}_{2}$ recovery kinetics), also present better performance in an intermittent specific test, which represents timeto-recovery between sprints and the ability to maintain sprint performance during highintensity intermittent exercise. These results may contribute to female athletes of various intermittent modalities to focus on training and improving their oxygen uptake off-transient kinetics in order to obtain better performance in this specific exercise modality. With such a perspective, this shows once more the importance of $\mathrm{VO}_{2}$ off-transient kinetics in the ability to maintain repeated-sprint performance during an intermittent modality training session or a match situation. Coaches and training staff could make use of regular aerobic capacity assessment of their athletes to monitor and design training strategies towards intermittent performance. 


\section{Acknowledgements}

The authors gratefully acknowledge all athletes for their enthusiastic participation, and laboratory colleagues. The results of the present study do not constitute endorsement of the product by the authors or the NSCA. The authors report no conflicts of interest.

\section{References}

Allen DG, Lamb GD, Westerblad H. Skeletal Muscle Fatigue: Cellular Mechanisms. Physiol Rev, 2008; 88: $287-332$

Aziz AR, Chia M, Teh KC. The relationship between maximal oxygen uptake and repeated sprint performance indices in field hockey and soccer players. J Sports Med Phys Fitness, 2000; 40: 195-200

Bailey SJ, Wilkerson DP, Dimenna FJ, Jones AM. Influence of repeated sprint training on pulmonary O2 uptake and muscle deoxygenation kinetics in humans. J Appl Physiol, 2009; 106: 1875-1887

Barstow TJ, Jones AM, Nguyen PH, Casaburi R. Influence of muscle fiber type and pedal frequency on oxygen uptake kinetics of heavy exercise. J Appl Physiol, 1996; 81: 1642-1650

Beltrame T, Hughson RL. Linear and non-linear contributions to oxygen transport and utilization during moderate random exercise in humans. Exp Physiol, 2017; 102: 563-577

Bishop D, Spencer M. Determinants of repeated-sprint ability in well-trained team-sport athletes and endurance-trained athletes. J Sports Med Phys Fitness, 2004; 44: 1-7

Bishop DJ, Girard O, Mendez-Villaneuva A, Mendez-Villanueva A. Repeated-sprint ability - part II: recommendations for training. Sport Med, 2011; 41: 741-756

Bogdanis GC, Nevill ME, Boobis LH, Lakomy HK, Nevill AM. Recovery of power output and muscle metabolites following $30 \mathrm{~s}$ of maximal sprint cycling in man. J Physiol, 1995; 482: 467-480

Børsheim E, Bahr R. Effect of exercise intensity, duration and mode on post-exercise oxygen consumption. Sport Med, 2003; 33: 1037-1060

Datson N, Drust B, Weston M, Gregson W. Repeated high-speed running in elite female soccer players during international competition. Sci Med Footb, 2019; 3: 150-156

Di Salvo V, Gregson W, Atkinson G, Tordoff P, Drust B. Analysis of high intensity activity in premier league soccer. Int J Sports Med, 2009; 30: 205-212

Dupont G, McCall A, Prieur F, Millet GP, Berthoin S. Faster oxygen uptake kinetics during recovery is related to better repeated sprinting ability. Eur J Appl Physiol, 2010; 110: 627-634

Dupont G, Millet GP, Guinhouya C, Berthoin S. Relationship between oxygen uptake kinetics and performance in repeated running sprints. Eur J Appl Physiol, 2005; 95: 27-34

Esco MR, Snarr RL, Flatt A, Leatherwood M, Whittaker A. Tracking changes in maximal oxygen consumption with the heart rate index in female collegiate soccer players. J Hum Kinet, 2014; 42: 103111

Evans JD. Straightforward statistics for the behavioral sciences. Brooks/Cole Publishing, Pacific Grove, CA; 1996

Glaister M. Multiple-sprint work: Methodological, physiological, and experimental issues. Int J Sports Physiol Perform, 2008; 3: 107-112

Hagberg JM, Hickson RC, Ehsani AA, Holloszy JO. Faster adjustment to and recovery from submaximal exercise in the trained state. J Appl Physiol, 1980; 48: 218-224

Harris RC, Edwards RH, Hultman E, Nordesjö LO, Nylind B, Sahlin K. The time course of phosphorylcreatine resynthesis during recovery of the quadriceps muscle in man. Pflugers Arch, 1976; 367: 137-142

Haseler LJ, Hogan MC, Richardson RS. Skeletal muscle phosphocreatine recovery in exercise-trained humans is dependent on O2 availability. J Appl Physiol, 1999; 86: 2013-2018

Helgerud J, Engen LC, Wisløff U, Hoff J. Aerobic endurance training improves soccer performance. Med Sci Sport Exerc, 2001; 33: 1925-1931

Impellizzeri FM, Rampinini E, Castagna C, Bishop D, Ferrari Bravo D, Tibaudi A, Wisloff U. Validity of a repeated-sprint test for football. Int J Sports Med, 2008; 29: 899-905 
Jones AM, Burnley M. Oxygen uptake kinetics: an underappreciated determinant of exercise performance. Int J Sports Physiol Perform, 2009; 4: 524-532

Jones AM, Doust JH. A 1\% treadmill grade most accurately reflects the energetic cost of outdoor running. J Sports Sci, 1996; 14: 321-327

Keir DA, Robertson TC, Benson AP, Rossiter HB, Kowalchuk JM. The influence of metabolic and circulatory heterogeneity on the expression of pulmonary oxygen uptake kinetics in humans. Exp Physiol, 2016; 101: 176-192

Kilding AE, Winter EM, Fysh M. A comparison of pulmonary oxygen uptake kinetics in middle- and longdistance runners. Int J Sports Med, 2006; 27: 419-426

Kushmerick MJ, Meyer RA, Brown TR. Regulation of oxygen consumption in fast- and slow-twitch muscle. Am J Physiol Physiol, 1992; 263: C598-C606

Meyer RA, Brown TR, Kushmerick MJ. Phosphorus nuclear magnetic resonance of fast- and slow-twitch muscle. Am J Physiol Physiol, 1985; 248: C279-C287

Murias JM, Kowalchuk JM, Paterson DH. Speeding of VO2 kinetics with endurance training in old and young men is associated with improved matching of local $\mathrm{O} 2$ delivery to muscle $\mathrm{O} 2$ utilization. J Appl Physiol, 2010; 108: 913-922

Myers J, Kaminsky LA, Lima R, Christle JW, Ashley E, Arena R. A reference equation for normal standards for VO2 max: Analysis from the fitness registry and the importance of exercise national database (FRIEND Registry). Prog Cardiovasc Dis, 2017; 60: 21-29

Nordsborg N, Mohr M, Pedersen LD, Nielsen JJ, Langberg H, Bangsbo J. Muscle interstitial potassium kinetics during intense exhaustive exercise: effect of previous arm exercise. Am J Physiol Integr Comp Physiol, 2015; 285: R143-R148

Rampinini E, Bishop D, Marcora SM, Ferrari Bravo D, Sassi R, Impellizzeri FM. Validity of simple field tests as indicators of match-related physical performance in top-level professional soccer players. Int J Sports Med, 2007; 28: 228-235

Romer LM, Polkey MI. Exercise-induced respiratory muscle fatigue: implications for performance. J Appl Physiol, 2008; 104: 879-888

Rossiter HB, Ward SA, Howe FA, Kowalchuk JM, Griffiths JR, Whipp BJ. Dynamics of intramuscular 31PMRS P(i) peak splitting and the slow components of PCr and O2 uptake during exercise. J Appl Physiol 2002; 93: 2059-2069

Ruby BC, Robergs RA. Gender differences in substrate utilisation during exercise. Sport Med, 1994; 17: 393410

Taylor J, Macpherson T, Spears I, Weston M. The effects of repeated-sprint training on field-based fitness measures: A meta-analysis of controlled and non-controlled trials. Sport Med, 2015; 45: 881-891

Taylor JM, Macpherson TW, Spears IR, Weston M. Repeated sprints: An independent not dependent variable. Int J Sports Physiol Perform, 2016; 11: 693-696

Weisman IM et al. ATS/ACCP Statement on cardiopulmonary exercise testing. Am J Respir Crit Care Med, 2003; 167: 211-277

Welch JF, Archiza B, Guenette JA, West CR, Sheel AW. Sex differences in diaphragmatic fatigue: the cardiovascular response to inspiratory resistance. J Physiol, 2018; 596: 4017-4032

Wragg CB, Maxwell NS, Doust JH. Evaluation of the reliability and validity of a soccer-specific field test of repeated sprint ability. Eur J Appl Physiol, 2000; 83: 77-83

\section{Corresponding author:}

\section{Audrey Borghi-Silva, Ph.D.}

Department of Physical Therapy. Federal University of São Carlos.

Rod. Washington Luis, km 235 - SP-310. CEP: 13565-905. São Carlos, São Paulo, Brazil

Tel: +55 (16) 3351 8952. Fax: +55 (16) 33612081

E-mail: audrey@ufscar.br 